\title{
Image Adaptive Edge Detection Based on Canny Operator and Multiwavelet Denoising
}

\author{
Lin Zhang \\ Jiangxi Science \& Technology Normal University, Nanchang, China \\ zymm_nc@163.com
}

Keywords: Edge Detection, Canny Operator, Multiwavelet Denoising, Adaptive Threshold

\begin{abstract}
Aim to problems of traditional canny operator, we propose an improved canny edge detection method. First, in order to overcome excessive smoothing, we use multiwave adaptive denoising method to instead of Gaussian filter. Then after obtaining non-maxima suppression image, we use mean values of gradient of entire image to adaptively select dual-threshold to instead of manually setting threshold. Our experiments are conducted on original image and noise image respectively. Experiments show compared with other traditional methods, this method can effectively remove noise, reduce the occurrence of pseudo-edge and obtain a more ideal edge detection effect finally.
\end{abstract}

\section{Introduction}

Edge is the boundary between the object need to be extracted and background, in order to separate the object and background, we have to detect and extract accurate edge. Separating the object and background has great practical significance for subsequent processing in many applications such as face recognition, image segmentation, image enhancement. Therefore, how to effectively improve the accuracy of edge detection is very worthy of study. Traditional edge detection operator has Roberts operator, Sobel operator, Prewitt operator, LOG operator and so on. These operators are local window gradient operator which have advantage of simple structure and fast speed. But because they are more sensitive to noise [1], the effect of them is not very satisfactory in the practical application.

In 1986, John Canny proposed canny criterion and on the basis of this criterion proposed optimal edge detection operator---canny operator [2]. This algorithm is based on the optimization algorithm which has advantages of big SNR and high detection progress. Experiments demonstrate that canny operator is superior to other traditional edge detection operators in terms of handling image polluted by Gaussian white noise [3]. Nevertheless, traditional canny operator still has its own drawbacks. Firstly, canny operator using Gaussian filter to remove noise is easy to result in the loss of edge information of the original image due to excessive smoothing. Secondly, high and low thresholds of canny operator need to manually set with experience. If selected threshold is too high which is easy to make the resulting edge discontinuous and loses a lot of details, on the contrary, if selected threshold is too low which is easy to generate pseudo-edge and comes with a lot of noise.

Therefore, aim at above shortages of canny operator, this paper proposes an image edge detection algorithm based on adaptive multiwavelet denosing. Improved canny edge detection algorithm uses image adaptive denoising algorithm based on multiwavelet we proposed in the literature [4] to instead of Gaussian filter to remove the noise, and then uses mean values of gradient of entire image method proposed in this paper to adaptively select dual-threshold. Experiments show that improved method can effectively remove noise in the image, adaptively select suitable dual-threshold and detect better edge.

The remaining section of this paper is organized as follows. Section 2 introduces traditional canny operator. Section 3 gives a detailed description of improved canny operator. Section 4 shows experimental results and analyses. Finally, conclusions are given in Section 5. 


\section{Canny Edge Detection Operator}

Canny Criterion. Canny gave three criterions for evaluating edge detection performance in the study of edge detection algorithm [3,5]:

(1) High SNR criterion: focus on good detection results which makes the probability of missing the true edge and false detection non-edge as small as possible

(2) High positioning accuracy criterion: distance of the detected edge point and the actual edge point is the nearest which makes edge positioning accuracy highest

(3) Single-edge response criterion: has only one response to single edge point which maximally inhibits the occurrence of pseudo-edge

Traditional Canny Operator. Canny operator includes four sections [3.5.6.7]: Gaussian smoothing filter, calculating the magnitude and direction of the gradient, non-maxima suppression and dual-threshold method for detecting and connecting edge.

(1) Gaussian smoothing filter

Canny operator selects two-dimensional Gaussian distribution function for image smoothing filter:

$$
G(x, y)=\frac{1}{2 \pi \sigma^{2}} \exp \left[-\frac{x^{2}+y^{2}}{2 \sigma^{2}}\right]
$$

where $\sigma$ is standard deviation of Gaussian function which is used to control the degree of smoothing. Convolution of filter template and the original image can realize smoothing image:

$$
I(x, y)=G(x, y) * f(x, y)
$$

(2) Calculating the magnitude and direction of the gradient

Calculating gradient can be achieved by partial derivative. Canny operator adopts finite difference of first-order partial derivate to calculate the magnitude $M(i, j)$ and direction $\theta(i, j)$ of the gradient:

$$
\begin{aligned}
& M(i, j)=\sqrt{f_{x}^{2}(i, j)+f_{y}^{2}(i, j)} \\
& \theta(i, j)=\arctan \frac{f_{y}(i, j)}{f_{x}(i, j)}
\end{aligned}
$$

Where $f_{x}(i, j)$ and $f_{y}(i, j)$ are the partial derivative of the $\mathrm{x}$ and $\mathrm{y}$ directions respectively, $M(i, j)$ reflects the edge strength of image and $\theta(i, j)$ reflects direction of the edge.

(3) Non-maxima suppression

Compare each pixel and two adjacent pixels along the gradient direction, if gradient magnitude of the pixel is larger than two adjacent pixel, then the pixel is determined as possible edge point, otherwise gradient magnitude of the pixel is set to 0 .

(4) Dual-threshold method for detecting and connecting edge

Canny operator adopts dual-threshold method to detect and connect the final edge from possible edge points. Dual-threshold includes a high threshold and a low threshold which has a certain corresponding relationship: low threshold $=0.4 *$ high threshold. Pixel gray value is set to 0 if gradient magnitude is small than high threshold to get image one, pixel gray value is set to 0 if gradient magnitude is small than low threshold to get image two. Image one can remove most of noise but lose a lot of edge information, image two retains more detail information but comes with a lot of noise. Therefore, image one is used as basis and image two is used as supplement to connect edge. So selecting suitable threshold is the key of canny operator.

\section{Improved Canny Edge Detection Operator}

Traditional canny operator using Gaussian filter to remove noise is easy to result in the loss of edge information due to excessive smoothing. Therefore, we use multiwavelet adaptive denoising 
method to instead of Gaussian filter in improved method. In this method, we proposed an adaptive denoising threshold which fully considers characteristics of the image itself [4]:

$$
\lambda=c \times \sigma \times \sqrt{2 \ln N} / 2^{j-1}
$$

Where $\sigma$ is noise standard deviation, $j$ is decomposition scale. We determine value of $c$ via genetic algorithm to get the best denoising effect. In traditional canny operator, high and low thresholds of canny operator need to manually set with experience which does not consider characteristics of the image itself and likely to cause bad effect of edge detection. Therefore, we propose mean values of gradient of entire image method to adaptively select dual-threshold. First, this method calculates gradient of entire image, and then calculates mean of gradient of all pixels of entire image. Finally, the mean is selected as high threshold, and low threshold is determined by a certain corresponding relationship mentioned above. Implementation process of improved canny operator is shown in Fig. 1.

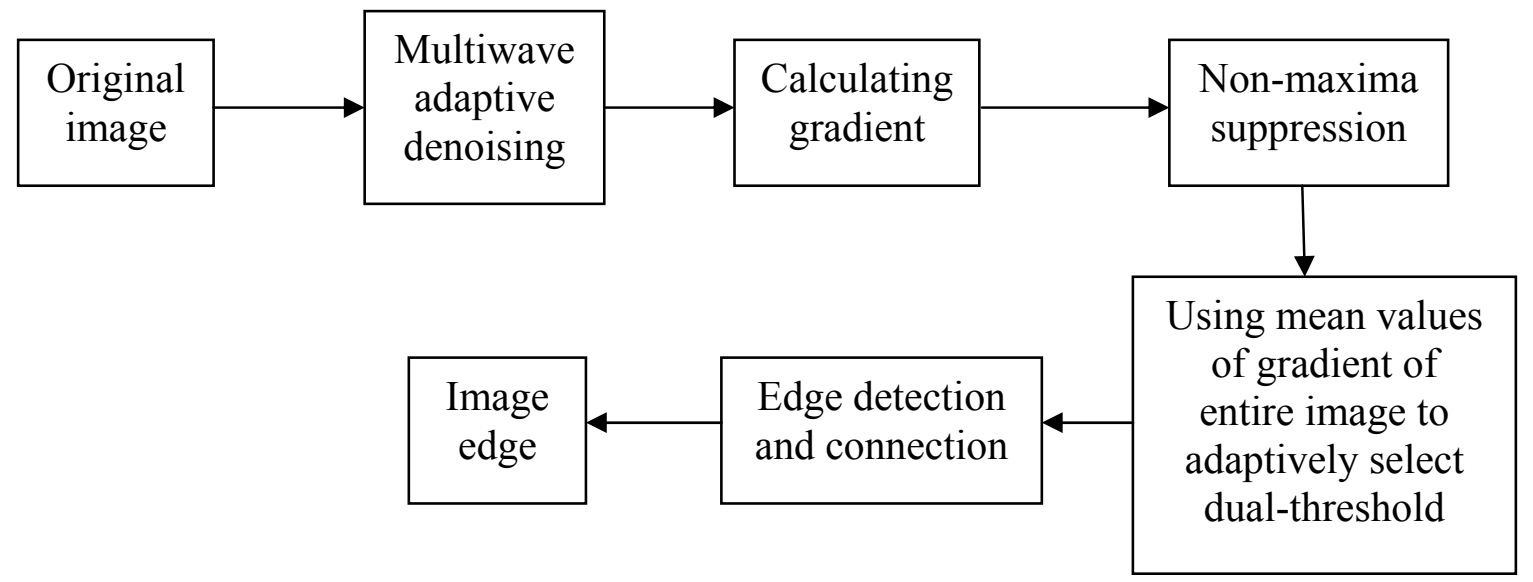

Fig. 1. Flow chart of improved canny operator

\section{Experimental Results and Analyses}

In our experiments, we use $256 * 256$ standard image "Lenna" as test image. In order to verify the effect of improved edge detection method, we compare improved method with traditional edge detection operators including Sobel, Roberts, LOG, prewitt and canny. Experiments are performed in both cases: no noise environment and noisy environment. Edge detection results of different method are shown in Fig.2 and Fig.3. Results of Fig.2 are in no noise environment. Results of Fig.3 are in the noisy environment with $\mathrm{SNR}=3$ Gaussian white noise. We can know from Fig. 2 that traditional canny operator is superior to other traditional operators. But it still exists a small part of discontinuity and pseudo-edge. The continuity of the detected edge of improved canny operator is the best. Fig. 3 shows that traditional canny operator is better than other traditional operators in terms of noise suppression. But it still detects many interference edge and noise points. Improved canny operator not only can accurately extract the edge, but also can effectively remove unnecessary interference of details and noise points.

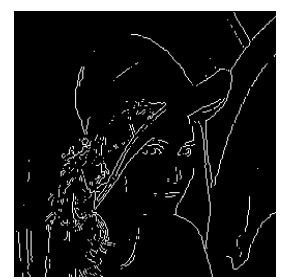

(a)

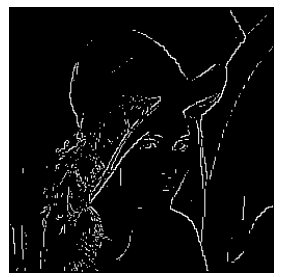

(b)

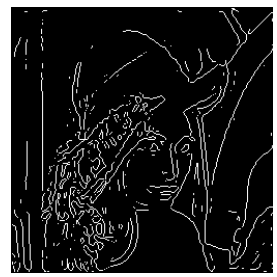

(c)

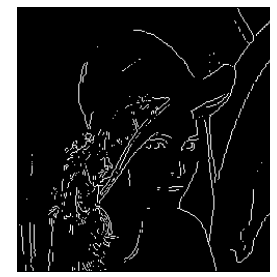

(d)

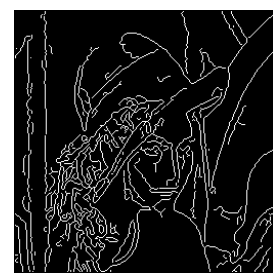

(e)

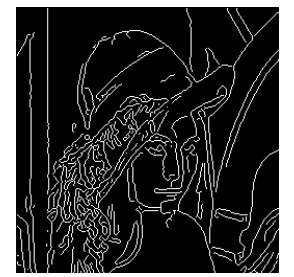

(f)

Fig. 2. Edge detection results of different in no noise environment: (a) Sobel; (b) Roberts; (c) LOG;

(d) prewitt; (e) canny; (f) improved canny 


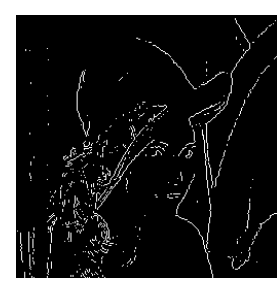

(a)

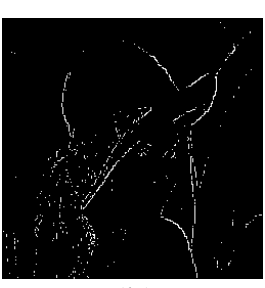

(b)

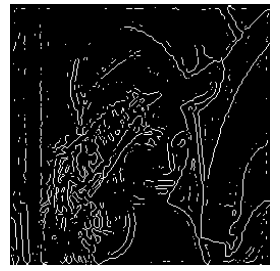

(c)

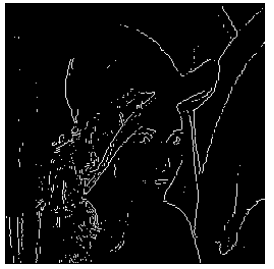

(d)

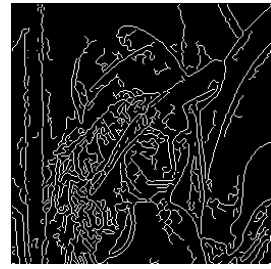

(e)

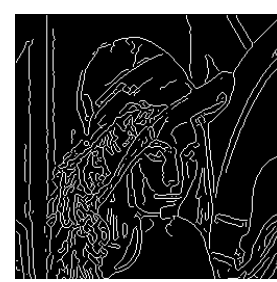

(f)

Fig. 3. Edge detection results of different in noisy environment: (a) Sobel; (b) Roberts; (c) LOG; (d) prewitt; (e) canny; (f) improved canny

\section{Conclusions}

Canny operator is currently the most widely used edge detection operator. Nevertheless, canny operator still has some shortcomings such as edge discontinuous, poor anti-noise ability and threshold lack of adaptability. Therefore, in this paper, we proposed an improved canny edge detection method. This method uses multiwave adaptive denoising method to effectively remove noise, adopts mean values of gradient of entire image to improve dual-threshold selection method which fully considers characteristics of the image. Experiments demonstrate that new method proposed in this paper can better suppress the effect of noise on edge detection, retain edge information very well and has a high positioning accuracy of detection.

\section{References}

[1] Y. Zhao, B.L. Zhou and H.X. Chen: Journal of Jilin University (Science Edition) Vol.50 (2012), p. $740-744$

[2] Z.Q. Zhang, H.S. Song: Computer \&Digital Engineering Vol.42 (2014), p.122-128

[3] X.J. Wang, X.M. Liu and Y. Guan: Computer Engineering Vol.38 (2012), p.196-198

[4] L. Zhang, Z.J. Fang, S.Q. Wang, F. Yang and G.D. Liu: J. Infrared Millim. Waves Vol.28 (2009), p.77-80

[5] C.C. Shang, H.W. Ma and J.Y. An: Computer Engineering \& Science Vol.36 (2014), p. 491-496

[6] Z.S. Zhang, J.Q. Xi and Y. Liu: Computer Science Vol.40 (2013), p.279-282

[7] H. Hu, J.H. He and X.Q. He: Computer Engineering and Applications Vol.50 (2014), p.161-165 